\title{
Extreme-precision measurements of cosmic rays via radio detection with the SKA
}

\author{
Anne Zilles ${ }^{1,2}$, Stijn Buitink ${ }^{3}$ and Tim Huege ${ }^{* 4}$ \\ ${ }^{1}$ EKP, Karlsruher Institut für Technologie, Kaiserstr. 12, 76131 Karlsruhe, Germany \\ 2 Sorbonne Universités, UPMC Univ Paris 6 et CNRS, UMR 7095, Institut d'Astrophysique de \\ Paris, 98 bis bd Arago, 75014 Paris, France \\ ${ }^{3}$ Astrophysical Institute, Vrije Universiteit Brussel, Pleinlaan 2, 1050 Brussels, Belgium \\ ${ }^{4}$ IKP, Karlsruher Institut für Technologie, Postfach 3640, 76021 Karlsruhe, Germany \\ E-mail: zilles@iap.fr, stijn.buitink@vub.ac.be, tim.huegeakit.edu
}

\begin{abstract}
The future SKA-low will provide an extremely dense and very homogeneous antenna array on an area of roughly $0.5 \mathrm{~km}^{2}$ and with a large bandwidth of $50-350 \mathrm{MHz}$. With minor engineering changes it would be ideally suited to detect extensive air showers initiated by cosmic rays in the Earth's atmosphere via their pulsed radio emission. The very precise measurements of individual cosmic-ray air-shower events allows detailed studies of the mass composition in the energy range around $10^{17} \mathrm{eV}$ where the transition from a galactic to an extragalactic origin could occur. We present a simulation study on cosmic-ray detection with focus on the potential to reconstruct the depth of shower maximum for individual showers to be measured with SKA-low, resulting in an expected intrinsic uncertainty on the Xmax reconstruction of less than $10 \mathrm{~g} / \mathrm{cm}^{2}$. We will discuss the dependencies of the uncertainty on the direction and energy of the primary as well as the parameters of the antenna array, e.g. the analysed bandwidth and antenna density.
\end{abstract}

35th International Cosmic Ray Conference

10-20 July, 2017

Bexco, Busan, Korea

* Speaker. 


\section{Introduction}

In the past decades, radio detection of cosmic-ray air showers has made huge progress [1]. It can provide additional information to classical cosmic-ray observation techniques. One question which can be addressed is the mass composition of cosmic rays in the energy range of the transition from Galactic to Extragalactic origin. Since the radio emission from particle showers contains the information of the complete longitudinal air-shower development, it is possible to reconstruct the shower maximum, a parameter which is correlated to the mass of the primary cosmic ray. The current best measurements of $X_{\max }$, achieving a resolution of $20 \mathrm{~g} / \mathrm{cm}^{2}$, are performed by fluorescenceand Cherenkov-light detection (see e.g. [2]).

The radio-antenna array LOFAR acts not just as a pathfinder for SKA, it also was enabled to perform cosmic-ray detection. The impressive mean $X_{\max }$ resolution of about $17 \mathrm{~g} / \mathrm{cm}^{2}$ [3], obtained for the reconstruction of the shower maximum in the energy range of $10^{17}-10^{17.5} \mathrm{eV}$, gives an indication of the potential for cosmic ray detection with the future SKA-low. This resolution was achieved by using a top-down analysis approach where each air-shower measurement is compared to a large number of simulated air showers induced by protons and iron-nuclei as primaries.

In this contribution, we present a simulation study and its results on the potential of SKA-low to perform ultra-precise air-shower measurements on the depth of the shower maximum, demonstrating that a a reconstruction of the shower depth $X_{\max }$ of individual showers with a mean intrinsic uncertainty of well below $10 \mathrm{~g} / \mathrm{cm}^{2}$ seems feasible.

The low-frequency core of the future Square Kilometre Array (SKA-low) will be located in West-Australia. It will consist of almost 70.000 antennas on an area of less than $0.5 \mathrm{~km}^{2}$. The dual-polarised antennas will be sensitive to frequencies from $50-350 \mathrm{MHz}$. Phase 1 of the lowfrequency part of SKA (SKA-low) will be built as of 2018 with the planned completion in 2023. The first operation is planned for 2020 while cosmic-ray measurements could already start earlier. Some technical design modifications in the data-acquisition system and the deployment of a simple particle-detector array for triggering are necessary to enable SKA-low for cosmic ray detection. More details can be found in [4]. Information on the preliminary design of the SKA-low core, which we include in this study, can be found in the configuration report by the SKA organisation [5].

\section{Reconstruction of the shower maximum and its dependencies}

Reconstruction method: The method for the reconstruction of the shower depth of individual detected showers we present in this contribution was developed and already successfully applied in the context of cosmic ray detection with LOFAR [3]. As basis for the simulation study to determine the possible uncertainty on the shower-maximum reconstruction, we prepared sets of air-shower simulations, induced by 50 protons and 25 iron nuclei for each energy and zenith angle. We performed the air shower simulation with CORSIKA [6] while the corresponding radio signal of the shower was calculated by CoREAS [7], a plug-in of CORSIKA. We chose 160 antenna positions as observers for which the radio signal is calculated in the shower plane defined by $\mathbf{v} \times \mathbf{B}$ and $\mathbf{v} \times(\mathbf{v} \times \mathbf{B})$, the so-called star-shape pattern, as shown in fig. 1 (left). The vector $\mathbf{v}$ represents the velocity of the air-shower front, $\mathbf{B}$ the Earth's magnetic field. For each position in the starshape pattern, the East-West and the North-South components of the electric field were filtered to 

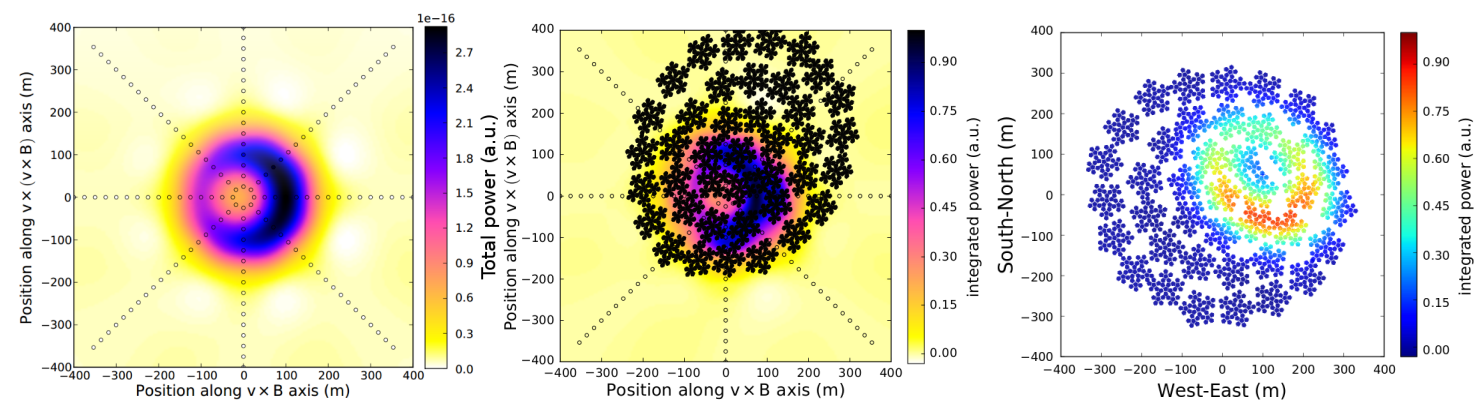

Figure 1: Left: Total over time integrated power at the simulated antenna positions (circles) and the interpolated radio footprint in the frame of the shower plane, defined by the axes $\mathbf{v} \times \mathbf{B}$ and $\mathbf{v} \times(\mathbf{v} \times \mathbf{B})$. Shown here is the simulated result for a proton-induced air shower with a primary energy of $E=10^{17} \mathrm{eV}$ and a zenith angle of $36.84^{\circ}$, filtered to a frequency band of $50-350 \mathrm{MHz}$. Center: The SKA-low antenna positions rotated into the shower plane (power normalised to the maximum value). At each antenna position, a value for the total received power is interpolated. Right: The example radio footprint on ground as detected by the SKA-low array, with North pointing upwards. The different colour scheme is chosen to underline the change of the coordinate system. Reprinted from [8].

the frequency band of $50-350 \mathrm{MHz}$. An antenna model was not included. Afterwards, the time integral of the total received power was computed for each antenna. The footprint of the radio signal can be reconstructed by interpolation between the rays of the star-shape pattern (see fig. 1, left), including also its asymmetry due to the interference of the Askaryan and the geomagnetic effect. To estimate the power received by the SKA-low antennas, the complete antenna array is rotated into the shower-plane frame and each antenna is assigned a total power corresponding to the interpolated value at its position (see fig. 1, center). This procedure is applied to every simulation in a set. Finally, each simulation acts once as "fake" data with random noise added to the interpolated total power of each antenna: $5 \%$ of the total power of each antenna as uncertainty on the antenna calibration and $1 \%$ of the maximum power in the whole array as an approximation for Galactic noise. This approximation yields a noise scaling with the energy of the primary particle for the reconstruction. A more realistic assumption has to be used in future calculations. The remaining simulations of the set are then compared to the "fake" data on the basis of a $\chi^{2}$ fit:

$$
\chi^{2}=\sum_{\text {antennas }}\left(\frac{P_{\text {fake }}-P_{\text {sim }}}{\sigma_{\text {fake }}}\right)^{2}
$$

with $P_{\text {fake }}$ as the total power, integrated over time, of a single antenna in the "fake" data set, $\sigma_{\text {fake }}$ denoting the assumed uncertainty due to noise, and $P_{\text {sim }}$ as the simulated power for the same position. This fit returns a $\chi^{2}$ value for every comparison of the "fake" data to one of the remaining simulations in a set, to which one can then connect the corresponding $X_{\text {sim }}$ known from the simulation. This leads to a distribution to which a parabola function can be fitted. One example is shown in fig. 2 (left). The parabola fit returns the "reconstructed" shower depth $X_{\text {reco }}$ as the $X_{\max }$ value for which the parabola achieves its minimum. For this study, we chose the shower-core position on ground to be at $100 \mathrm{~m}$ east and $50 \mathrm{~m}$ north w.r.t. the center position of SKA-low, to guarantee a study of a general example of SKA-low 'contained' events as well as to avoid the effects of the radial symmetry of SKA-low. The absolute difference of the reconstructed shower maximum $X_{\text {reco }}$ to each "real" shower depth $X_{\text {real }}$ of the simulation which works as "fake" data is filled into a his- 

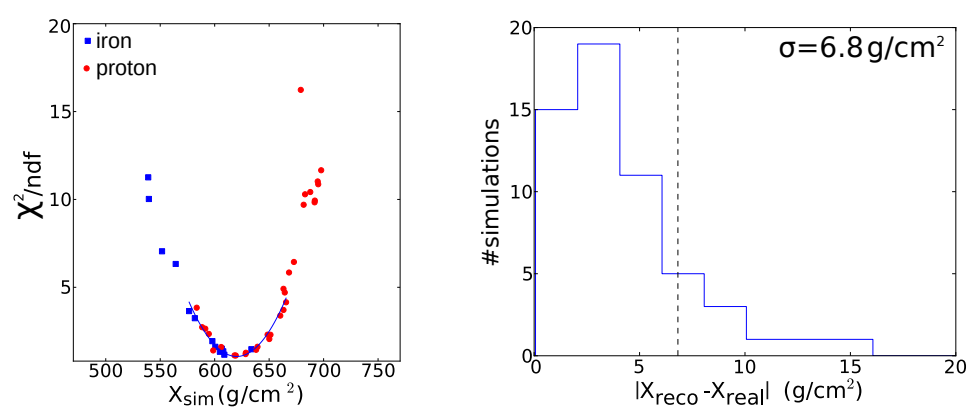

Figure 2: Left: An example $\chi^{2}$ distribution for the comparison of one "fake" event for SKA-low with the remaining simulations of a set with a primary of $E=10^{17} \mathrm{eV}$ and a zenith angle of $36.84^{\circ}$. Right: Histogram for the absolute differences of the reconstructed and the "real" shower depth for a complete simulation set with a primary energy of $E=10^{17} \mathrm{eV}$ and a zenith angle of $36.84^{\circ}$. Outliers beyond a difference of $20 \mathrm{~g} / \mathrm{cm}^{2}$ are not shown, but included in the calculations of the reconstruction uncertainty.

togram, as shown in Fig. 2 (right). In that histogram the $1 \sigma$ uncertainty is defined by the value of $\left|X_{\text {reco }}-X_{\text {real }}\right|$ which contains $68 \%$ of entries. The value is interpreted as the statistical uncertainty of the shower reconstruction by the method itself when adopting all simulations in a set once as a "fake" event. For the example given in fig. 2 (right), the resulting mean reconstruction uncertainty achieves a value of $6.8 \mathrm{~g} / \mathrm{cm}^{2}$ for a simulation set of 50 proton and 20 iron showers with a primary energy of $E=10^{17} \mathrm{eV}$, an azimuth angle of $225^{\circ}$ and a zenith angle of $36.84^{\circ}$.

The impact of the antenna density: In this simulation study only every 8th antenna of the SKA-low array is included in the comparison to simulations, which can be also interpreted as a reduced number of antennas read out for an air-shower event or a reduced antenna density w.r.t. the whole SKA-low antenna array. To prove that this has no significant impact on the results, we include in the applied reconstruction method all SKA-low antennas for the same simulation set of 50 proton and 20 iron-induced air showers. The results are shown in fig. 3 (left). Since the background noise is randomly added to the simulated signal, the histogram is slightly different compared to fig. 2. Nevertheless, the resulting uncertainty for the depth of the shower maximum of $6.8 \mathrm{~g} / \mathrm{cm}^{2}$ has not changed when for including all SKA antennas. This shows that reducing the number of antennas in the reconstruction to every 8th antenna is valid and does not affect the result. However, we also studied the effect of a further thinning of the antenna array. A further thinning by including only every 16th antenna leads to an uncertainty of $7.2 \mathrm{~g} / \mathrm{cm}^{2}$ which is slightly worse than in the former result. Reading-out only every 32nd antenna as shown in fig. 3 increases the reconstruction uncertainty to a value of $7.4 \mathrm{~g} / \mathrm{cm}^{2}$. The results are summarised in Tab. 1. A further reduction of the numbers of antennas would lead to a much worse precision in the reconstruction. In general, reasonable thinning of the antenna array, i.e. just reading out a subset of the antennas, leads to a slightly worse reconstruction uncertainty, but does not affect the precision on the shower maximum reconstruction significantly as long as the read-out is homogeneously distributed over the radio footprint. Not to read out all antennas can therefore be used to reduce the amount of data which have to be transferred and analysed for the individual detected air-shower events. This study shows that SKA-low significantly profits from the high antenna density with which the radio footprint on the ground is sampled. However, the examination of the impact of the number of the read-out antennas is closely connected to and therefore limited by the number of simulated antenna 

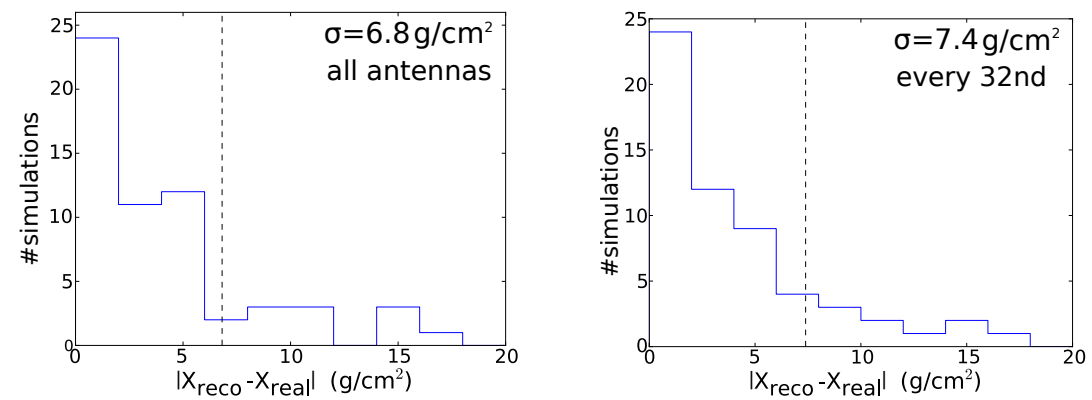

Figure 3: Histogram for the absolute differences of the reconstructed and the "real" shower depth for the air-shower detection with the SKA-low array. Left: including all antennas in the reconstruction, leading to a reconstruction uncertainty of $6.8 \mathrm{~g} / \mathrm{cm}^{2}$, right: including every 32nd antenna in the reconstruction, leading to a reconstruction uncertainty of $7.4 \mathrm{~g} / \mathrm{cm}^{2}$. Some outliers beyond a difference of $20 \mathrm{~g} / \mathrm{cm}^{2}$ are not shown but included in the calculations of the reconstruction uncertainty.

Table 1: Summary of the uncertainties of the reconstruction of the depth of the shower maximum in dependence on the antenna density for primary energy of $E=10^{17} \mathrm{eV}$ and a zenith angle of $36.84^{\circ}$.

\begin{tabular}{c|c|c|c} 
antenna thinning factor & mean antenna density & mean antenna distance & uncertainty \\
\hline \hline all & 0.16 ant. $/ \mathrm{m}^{2}$ & $2.5 \mathrm{~m}$ & $6.8 \mathrm{~g} / \mathrm{cm}^{2}$ \\
\hline every 8th & 0.02 ant. $/ \mathrm{m}^{2}$ & $7.0 \mathrm{~m}$ & $6.8 \mathrm{~g} / \mathrm{cm}^{2}$ \\
\hline every 16th & 0.01 ant. $/ \mathrm{m}^{2}$ & $10.0 \mathrm{~m}$ & $7.2 \mathrm{~g} / \mathrm{cm}^{2}$ \\
\hline every 32nd & 0.005 ant. $/ \mathrm{m}^{2}$ & $14.1 \mathrm{~m}$ & $7.4 \mathrm{~g} / \mathrm{cm}^{2}$
\end{tabular}

positions, whose impact has to be explored in another study.

Impact of the frequency band: Besides the much higher antenna density of SKA-low, the array differs from LOFAR especially in the frequency range it covers. While LOFAR measures in the frequency band of 30-80 MHz containing a large part of the radio energy [10], SKA-low covers frequencies from $50-350 \mathrm{MHz}$. The reconstruction of the shower maximum could therefore profit from the fact that for higher frequencies the structure of the Cherenkov cone becomes prominent. In the frequency band of $30-80 \mathrm{MHz}$, a bean-like structure is visible in the radio footprint (see fig. 4, left) instead of a clear Cherenkov ring as for the higher frequencies as for the frequency band of $50-350 \mathrm{MHz}$. It is observable that the received power integrated over time in the frequency band of $50-350 \mathrm{MHz}$ is higher than the one at $30-80 \mathrm{MHz}$. In addition, the galactic noise level drops at higher frequencies so that a separation from noise will be easier. For the same simulation set and antenna density as used for the results in fig. 2, we obtain a much larger reconstruction uncertainty of $16.5 \mathrm{~g} / \mathrm{cm}^{2}$ for the depth of the shower maximum when filtering the signal to $30-$ $80 \mathrm{MHz}$, as illustrated in Fig. 4 (right). In comparison to $50-350 \mathrm{MHz}$, the parabola for $30-$ $80 \mathrm{MHz}$ is wider so that the minimum of the $\chi^{2}$ distribution is less accurately defined (compare fig. 4 (center) and fig. 2 (left)). This leads to the conclusion that besides a received power which is higher in the SKA-low frequency band, in comparison to LOFAR, SKA-low seems to profit for the $X_{\max }$ reconstruction from the measurements of the higher frequencies by the prominent Cherenkov cone structures. It has to be investigated in a future study whether additional parameters characterising the footprint can be found in the higher and larger frequency band which could be 

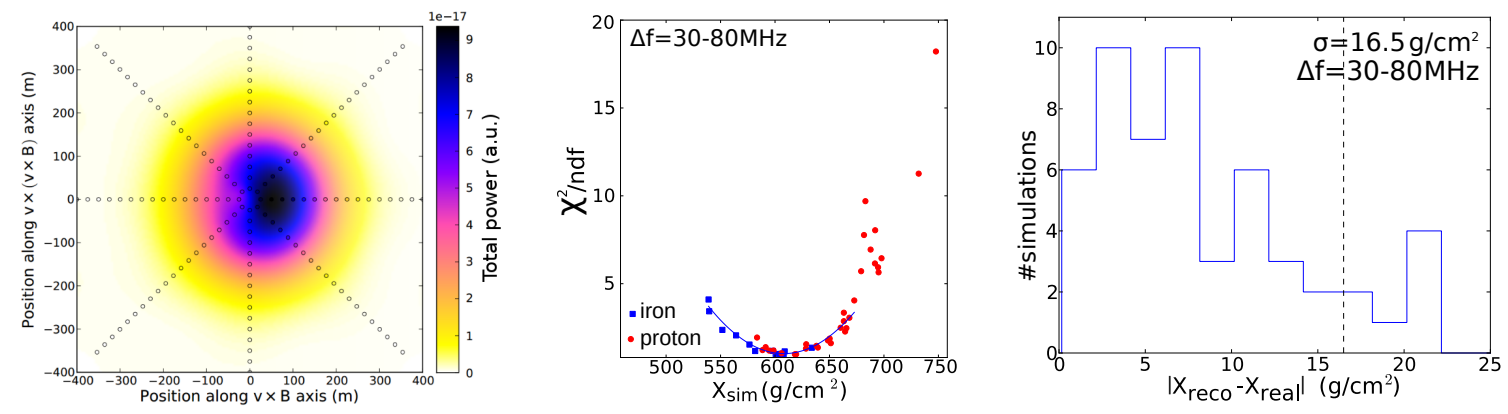

Figure 4: Left: Total integrated power at the simulated antenna positions (circles) and the interpolated radio footprint in the shower plane for a proton-induced air shower with a primary energy of $E=10^{17} \mathrm{eV}$ and a zenith angle of $36.84^{\circ}$, filtered to a frequency band of $30-80 \mathrm{MHz}$. Reprinted from [9]. Center: The $\chi^{2}$ distribution for the SKA-low array layout for a frequency band of $30-80 \mathrm{MHz}$. Right: Histogram for the absolute differences of the reconstructed and the "real" shower depth for the air-shower detection with the SKA-low array being sensitive to $30-80 \mathrm{MHz}$, leading to a reconstruction uncertainty of $16.5 \mathrm{~g} / \mathrm{cm}^{2}$.

Table 2: Summary of the uncertainties of the reconstruction of the depth of the shower maximum in dependence on the energy of the primary particle with a zenith angle of $36.84^{\circ}$.

\begin{tabular}{c|c|c|c|c|c} 
energy & $10^{16} \mathrm{eV}$ & $10^{16.5} \mathrm{eV}$ & $10^{17} \mathrm{eV}$ & $10^{17.5} \mathrm{eV}$ & $10^{18} \mathrm{eV}$ \\
\hline uncertainty & $9.8 \mathrm{~g} / \mathrm{cm}^{2}$ & $8.3 \mathrm{~g} / \mathrm{cm}^{2}$ & $6.8 \mathrm{~g} / \mathrm{cm}^{2}$ & $4.5 \mathrm{~g} / \mathrm{cm}^{2}$ & $3.5 \mathrm{~g} / \mathrm{cm}^{2}$
\end{tabular}

added as additional information to the existing method for $X_{\max }$ reconstruction.

Dependence on the primary energy: For a fixed zenith angle of $36.84^{\circ}$, we prepared a complete simulations set for different primary energies. Fig. 5 (left) shows the power distribution for one proton event in the shower plane for a primary energy of $E=10^{16} \mathrm{eV}$ and $E=10^{18} \mathrm{eV}$, respectively, which can be directly compared to fig. 1. It is observable that the radius of the Cherenkov ring, which becomes prominent in the radio footprint for $f>100 \mathrm{MHz}$ [1], depends on the primary energy, as expected from geometrical considerations according to the Heitler model: for air showers induced by lower energetic primaries the source of radiation is on average further away from ground than for air shower induced by higher energetic primaries, leading to a larger ring size. In addition, one can also notice that the power of the radio signal increases with rising energy of the primary particle emphasizing the narrow structure of the Cherenkov ring. The results, listed in Tab. 2, give evidence for a tendency of a decreasing reconstruction uncertainty with rising primary energy. This is not an effect of the rising signal-to-noise ratio since in this study the included noise scales (unrealistically) with the energy of the primary particle. In other words, the signal-to-noise ratio does not increase with higher primary energy. Indeed, for low energetic events this assumption could be too optimistic. The decreasing uncertainty could be explained by a possible better resolution of structures at the Cherenkov cone due to smaller geometrical distance and larger signals. Consequently, the more prominent the structures in the two dimensional profiles are, the better the simulation which fits best can be identified. The example $\chi^{2}$ distributions in the fig. 5 (bottom) support this theory. They are showing the trend that the jitter of the points around the parabola function decreases with higher energies. The uncertainty on the determined minimum decreases since the minimum of the parabola can be determined more precisely. For primary particles with 

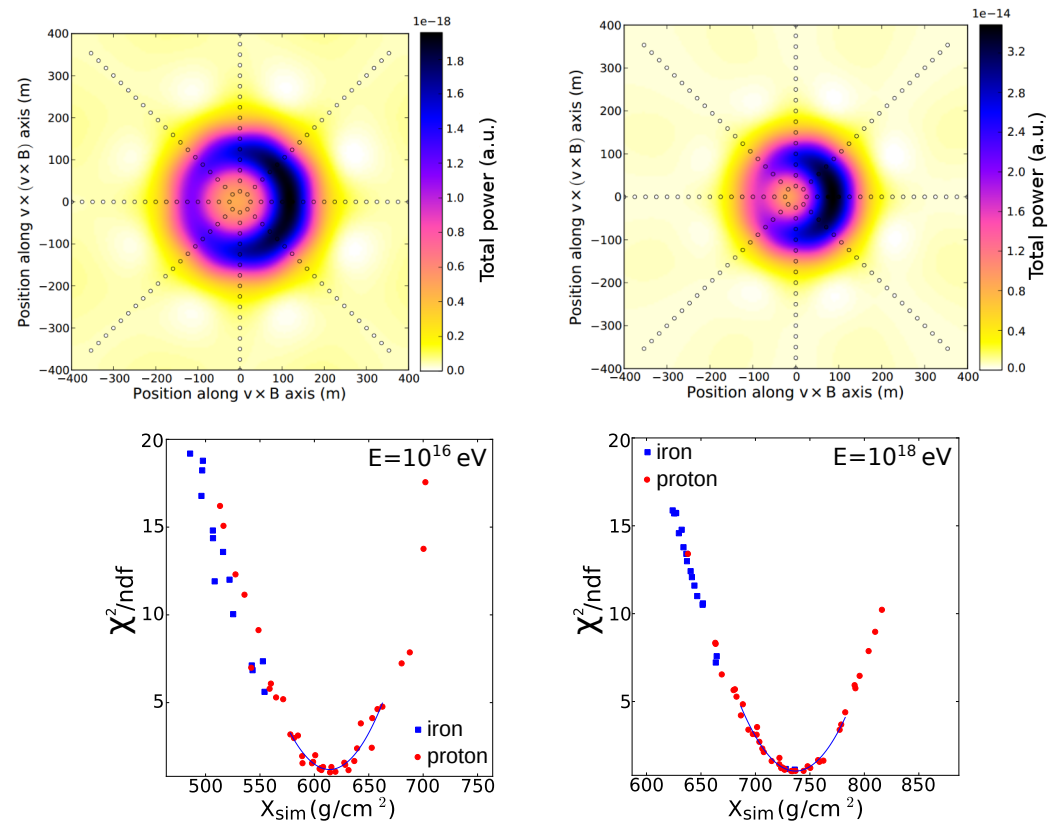

Figure 5: Top: Example simulated footprints of the radio signal in the shower plane for a proton-induced air shower, filtered to the frequency band of $50-350 \mathrm{MHz}$. Left: for a primary energy of $E=10^{16} \mathrm{eV}$, right: for a primary energy of $E=10^{18} \mathrm{eV}$. Reprinted from [9]. Bottom: Example $\chi^{2}$ distributions for a proton-induced air shower with different primary energies. Left: for a primary energy of $E=10^{16} \mathrm{eV}$, right: for a primary energy of $E=10^{18} \mathrm{eV}$.
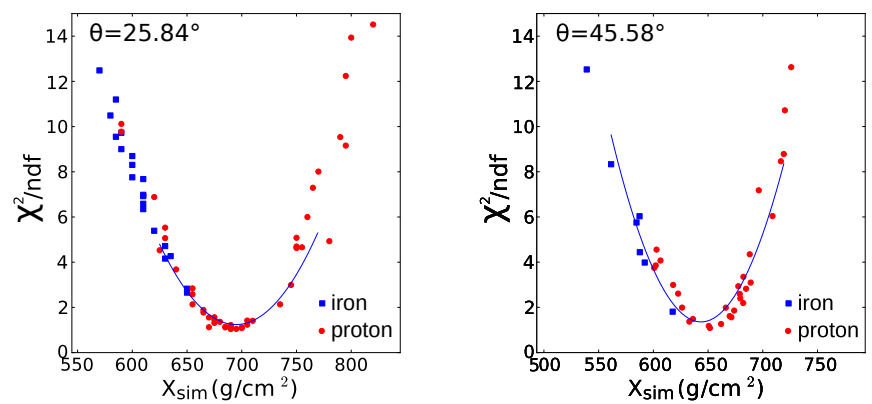

Figure 6: Example $\chi^{2}$ distribution for a proton-induced air shower with a primary energy of $E=10^{17} \mathrm{eV}$ and a zenith angle of $25.84^{\circ}$ (left) and of $45.58^{\circ}$ (right), respectively.

high energies, SKA-low will be able to reconstruct the depth of the shower maximum on individual air shower events extremely precisely.

Dependence on the zenith angle: In the following, we study the impact of the zenith angle on the reconstruction uncertainty. We produced complete simulation sets for a primary energy of $E=10^{17} \mathrm{eV}$ and a zenith angle of $25.84^{\circ}$ and $45.58^{\circ}$ which will be compared to the previous results gathered at $36.84^{\circ}$. Fig. 6 displays a zoom in the $\chi^{2}$ distributions $\left( \pm 150 \mathrm{~g} / \mathrm{cm}^{2}\right.$ around the minimum) for example events with a zenith angle of $25.84^{\circ}$ and $45.58^{\circ}$, respectively. The comparison of the plots shows that for the higher zenith angle fewer simulations lead to a small $\chi^{2} /$ ndf in the comparison to the "fake" data. In addition, the scatter of the points around the parabolic function gets larger. This could be explained geometrically: the source of the radio 
emission is further away from the ground for larger zenith angle so that the structure in the footprint get less prominent. Here, we have to set the previously chosen value of 5 for $\chi^{2} /$ ndf, below which the points are included in the parabola fit for finding the minimum, to 9 to ensure that for all comparisons a significant number of points are included in the fit. The result is that for the zenith angle of $25.84^{\circ}$ a reconstruction uncertainty of $6.1 \mathrm{~g} / \mathrm{cm}^{2}$ is achieved, while for the zenith angle of $45.58^{\circ}$ the reconstruction uncertainty is determined to $17.3 \mathrm{~g} / \mathrm{cm}^{2}$. The increase of the uncertainty could be originating in the decrease of the maximal power and the effect that the power distribution gets slightly broader as well as the asymmetry of the signal on the Cherenkov cone gets smaller for increasing zenith angles. A more refined approach in the reconstruction procedure could potentially achieve a significantly improved precision.

Another observation we made is that for the larger zenith angle a slight offset between the points of the proton and the iron induced shower appears in the $\chi^{2}$ distribution. The origin and whether this include additional information to distinguish between individual primary particles has yet to be studied.

\section{Conclusion}

Within this presented simulation study, we demonstrated that a LOFAR-like approach for the reconstruction of the shower depth for single events is applicable to SKA1-low data. We could show that SKA will be able to reconstruct the depth of the shower maximum on individual air shower events with an extreme precision of $10 \mathrm{~g} / \mathrm{cm}^{2}$, given by the reconstruction method itself, and therefore surpass classical detection techniques. We could demonstrate that the reconstruction will profit from the high antenna density while on the other hand an inclusion of just a subset of antennas into the reconstruction procedure, e.g. to reduce the amount of transferred data, will not affect the precision on the shower maximum reconstruction significantly as long as the antennas are homogeneously distributed over the radio footprint. In comparison to LOFAR, SKA-low seems to profit from the measurements at higher frequencies where the Cherenkov ring becomes the prominent pattern in the footprint. In a future study it has to be investigated whether additional parameters characterising the footprint can be found in the higher and larger frequency band which could be added as additional information to the existing method for $X_{\max }$ reconstruction. The influence of experimental uncertainties (realistic background noise, impact of the dynamic range, uncertainty in the atmospheric properties, ...) has to be evaluated as well.

\section{References}

[1] T. Huege, Physics Reports 620,1 (2016).

[2] A. Aab, P. Abreu, M. Aglietta, et al., Nucl. Instrum. Meth. A 798 , 172 (2015).

[3] S. Buitink et al., Nature 531 (2016) 70.

[4] T. Huege, J. D. Bray, S. Buitink et al., EPJ Web of Conferences, 135, 02003 (2017).

[5] SKA Organisation, http://astronomers.skatelescope.org/wp-content/uploads/2015/11/SKA1Low-Configuration_V4a.pdf (draft), 2015.

[6] D. Heck et al., Report FZKA 6019 (1998).

[7] T. Huege, M. Ludwig, \& C. James, AIP Conference Proceedings 1535, 128-132 (2012).

[8] A. Zilles, S. Buitink, \& T. Huege, EPJ Web of Conferences, 135, 02004 (2017).

[9] A. Zilles, PhD thesis: "Emission of Radio Waves in Particle Showers", Karlsruhe Institute of Technology (2016), to be published in Springer Theses, ISBN: 978-3-319-63410-4.

[10] P. Schellart et al., Astron. Astrophys. 560, A98 (2013). 